Polymer Journal, Vol. 38, No. 8, pp. 757-766 (2006)

(C) 2006 The Society of Polymer Science, Japan

\title{
Application of Catastrophe Theory to the Neck-initiation of Semicrystalline Polymers Induced by the Intercluster Links
}

\author{
Koh-hei NitTA ${ }^{1, \dagger}$ and Motowo TAKAYANAGI ${ }^{2}$ \\ ${ }^{1}$ Division of Material Sciences, Graduate School of Natural Science and Technology, \\ Kanazawa University, Kanazawa 920-1192, Japan \\ ${ }^{2}$ Emeritus Professor, Kyushu University
}

(Received January 13, 2006; Accepted March 23, 2006; Published June 30, 2006)

\begin{abstract}
This paper deals with the neck initiation in semi-crystalline polymers having lamellar clusters connected by the intercluster links composed of extended-chain bundles as demonstrated by electron microscope pictures of polyethylene and polypropylene. When semicrystalline polymers are uniaxially deformed at a constant rate, the necking is usually initiated and the necking zone propagates at a constant speed along the sample specimen. The necking initiation is explained in this paper by a first order catastrophic phase transition in analogy of the model of van der Waals gas. Catastrophic arrangement of the intercluster links results in sudden emergence of the locally oriented region which will be a seed of the neck formation in further deformation. The analysis succeeded in phenomenological indications of the critical condition determining whether the deformation is accompanied by neck or not. [doi:10.1295/polymj.PJ2005211]

KEY WORDS Catastrophe Theory / Neck Initiation / Semicrystalline Polymers / Intercrystalline Link /
\end{abstract}

Necking or cold drawing was apparently first observed by Carothers and Hill in 1932. ${ }^{1}$ The necking phenomena, i.e., formation of localized ordering within a uniaxially stretched specimen have been widely observed and studied in many polymeric systems. In semicrystalline polymers such as polyethylene (PE) and polypropylene (PP), necking is associated with large-scale morphological transformation from isotropic spherulitic to anisotropic fibril structures accompanied by the destruction and/or rearrangement of the parts of the crystals. The yield point appeared as the maximum point of the nominal stress $\sigma$-elongation $\lambda$ curves, accompanied by a lot of microshear bands. Beyond the yield point, the shear bands coalesce into a well-defined neck, the boundary of which propagates under essentially constant flow stress. ${ }^{2} \mathrm{Fi}-$ nally when the necking boundaries have propagated throughout the entire length of the specimen, the deformation will exhibit the character of strain hardening, and the specimen will break.

When the specimen is extended at conventional elongation speed, a rise of temperature occurs in the region of the neck. It has long been considered that heterogeneous deformations in necking are caused by the local adiabatic heating. However, Brauwer-Müller ${ }^{3}$ and Lazurkin ${ }^{4}$ found that necking still take place under quasi-static conditions at very slow extension rates. Vincent ${ }^{5}$ and Allison-Ward ${ }^{6}$ demonstrated that the increase in temperature is quite small and the adiabatic temperature is not sufficient to give an explana- tion for necking. Further studies showed that when a necked specimen is heated up to melting temperature, shrinkage is observed which finally results in a recovery of the shape before necking. This forced elasticity effects strongly suggests the existence of molecular networks which have been formed during crystallization of the isotropic sample and the structure of which seems not to be modified significantly during deformation. Detail discussion of these phenomena is found in the text by Strobl.

It is well known that the deformation of the sample is homogeneous up to the yield point ${ }^{8}$ whereas once a neck forms, the deformation is inhomogeneous and large strains are involved in the neck. According to the geometrical method using Considére construction, in which the $\sigma \lambda$ is plotted against $\lambda$, the initiation and stabilization of "neck" can be realized as maximum and minimum points. ${ }^{7,9}$ Hutchinson and Neale ${ }^{10}$ demonstrated that tensile neck process can be treated as a first order phase transition induced by force. Ericksen ${ }^{11}$ found a remarkable analogy between the necking process of a forced elastic bar and phase transition in the van der Waals gas. The equilibrium coexistence of unnecked and necked portions was determined by Maxwell rule devising the graphical method to identify equilibrium. The Ericksen theory was extended to a linear viscoelastic solid by Bernstein and Zappas. ${ }^{12}$ Recently, Leonov ${ }^{13}$ developed a theory on the based of a qualitative model by Gent et al. ${ }^{14,15}$ that necking is caused by transferring unfolding chains

${ }^{\dagger}$ To whom correspondence should be addressed (Tel: +81-76-234-4818, Fax: +81-76-264-6220, E-mail: nitta@ t.kanazawa-u.ac.jp). 

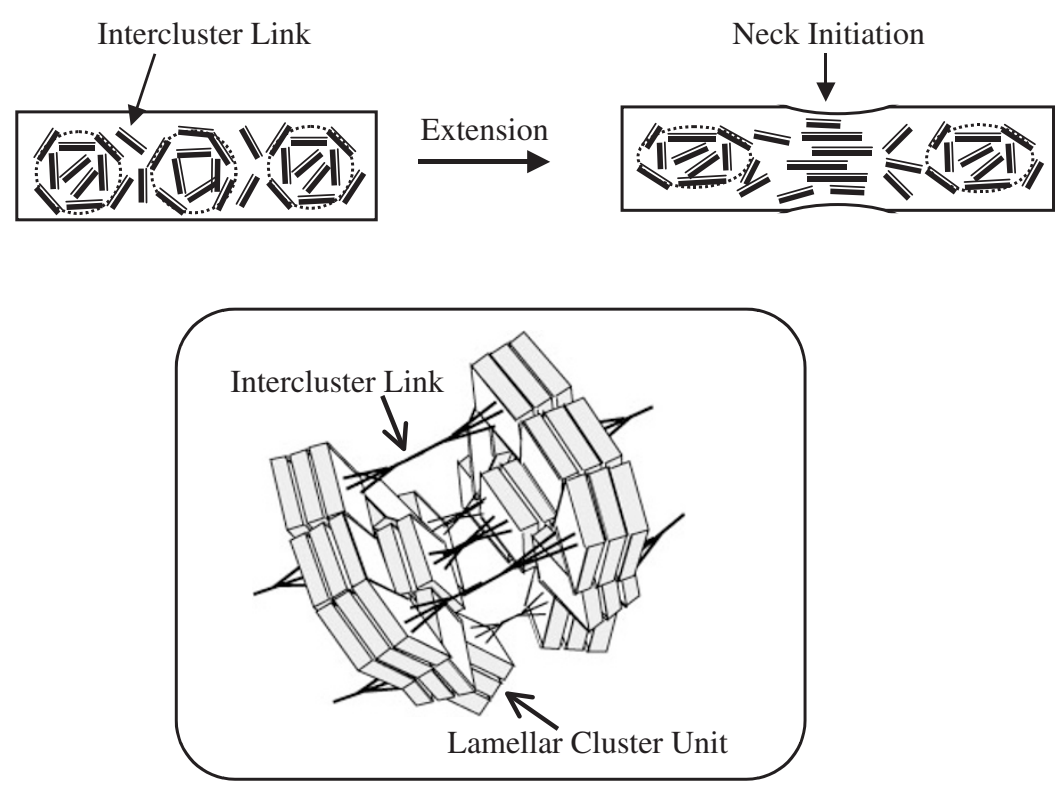

Figure 1. Schematic illustration of the neck nucleation process. The inset gives the sketch of the structural feature within deformed spherulites at yield point.

of crystalline blocks into amorphous network phase. Coleman-Newman ${ }^{16,17}$ numerically predicted the feature of neck propagation by introducing the reduced area portion in elastic and viscoelastic bars as an initial condition. Their simulation feature was finely in accordance with familiar observation of neck formation and propagation. However, there was a lack in fundamental understanding the neck initiation and stability.

In this work we focused on the neck initiation in semicrystalline polymers. So far, there are many excellent theoretical approaches for neck propagation and formation but few physical and mathematical models have been proposed for explaining why the area reduction or strain localization suddenly occurs during homogeneous deformation under a uniaxial tension. This spontaneous extended portion could be nuclei for the neck formation. This paper is focused on the initiation of the reduced area or the localization of strain in a uniform material under a uniaxial deformation.

\section{BACKGROUND}

This section contains a review of the background information needed for the theoretical treatment of the present paper.

Recently we have succeeded in explaining the emergences of intercrystalline links and piles of crystalline lamellae within spherulites shown in transmission electron-microscopic (TEM) pictures of $\mathrm{PE}^{18,19}$ and $\mathrm{PP}^{20}$ in terms of active and inactive tie-chains. According to our theoretical treatments, ${ }^{21}$ the inter- twining inactive tie-chains bind several stacked lamellae forming lamellar clusters and bundles of extended active tie-chains bridge adjacent lamellar clusters.

The active tie chains or intercluster links (ICL's) support the external force and their tractive forces act on the cluster surface, leading a portion of the lamellar cluster to be bent around one intercluster link. Further deformation leads to the fragmentation of lamellar clusters into the cluster units around ICL's at yield point (see the inset of Figure 1). The fragmentation of lamellar clusters releases the ICL's embedded in the lamellar clusters and makes it possible to cause the rearrangement of the decorated ICL units. Consequently, the decorated ICL units randomly disperse in the spherulitic matrix in the pre-yield region and further deformation leads to localized ordering of the ICL units above the yield point as shown in Figure 1. On the basis of this idea, this paper develops a mathematical model for an explanation of neck initiation. The inactive tie-chains could play a central role in the neck stabilization and propagation.

This structural feature of ICL units in the neck region is strongly supported from the TEM results by Kanig $^{22}$ that a network of fibers cross-linked by crystal blocks is formed on deformed PE films. Kilian et al. ${ }^{23-25}$ also identified the presence of stacks of several lamellae running parallel and forming clusters on the basis of TEM observations for a uniaxially deformed PE. Moreover they showed that the stressstrain curves of PE samples can be described using a van der Waals type of constitutive equation on the basis of the assumption that a cluster-network essentially governs deformation at high elongations. ${ }^{23}$ 


\section{THEORETICAL PART}

We here consider the constitutive relation for uniaxial deformation of semicrystalline polymers just after yielding. For the sake of simplicity, we further assume these polymers to be incompressible. As well known, the tensile data in the post-yielding region over the range of strain rate and temperature covered by usual tensile experiments are better fitted with an additive equation of the type $\mathrm{e}^{26-28}$

$$
\sigma(\lambda, \dot{\lambda} ; T)=\sigma_{p}(\lambda, \dot{\lambda} ; T)+\sigma_{e}(\lambda ; T)
$$

where $\lambda$ is the extensional ratio, $\lambda$ is the time derivative of $\lambda$, and $T$ is the absolute temperature. Here the mechanical quantities of stress and strain can be considered to be the deviatoric components because the hydrostatic component does not affect the yielding and/or plastic drawing process. The first term $\sigma_{p}$ represents the strain rate sensitivity of the material reflecting plastic flow of crystalline regions and the second term $\sigma_{e}$ reflects the elastic component of rubber networks. Leonov ${ }^{13}$ showed that the $\sigma_{p}$ is basically given by $G_{c}\left(1-\lambda^{-3}\right)$, which rapidly reaches a constant value with growing $\lambda$.

As well known, the plastic flow stress is nearly constant during neck propagation, and the $\sigma_{p}$ value has a negative dependence on temperature and a positive dependence on strain rate. These empirical characteristics of plastic flow stress can be expressed by Eyring rate process, ${ }^{29-31}$

$$
\sigma_{p}=\frac{\Delta H}{V^{*}}-\frac{R}{V^{*}} T \ln \frac{\dot{\varepsilon}_{0}}{2 \dot{\varepsilon}}
$$

where $V^{*}$ is the activation volume of sliding units, $\Delta H$ is the activation energy for plastic flow process, $\dot{\varepsilon}_{0}$ is a constant pre-exponential factor and $\dot{\varepsilon}$ is the strain rate. Consequently, the plastic component can be rewritten as

$$
\sigma_{p}=\gamma\left(T_{p}-T\right)
$$

where $\gamma=\frac{R}{V^{*}} \ln \frac{\dot{\varepsilon}_{0}}{2 \dot{\varepsilon}}$ and $T_{p}=\frac{\Delta H}{R}\left(\ln \frac{\dot{\varepsilon}_{0}}{2 \dot{\varepsilon}}\right)^{-1}$. It was confirmed that our experimental values of plateau stress in the post-yielding region for an isotactic PP can be fitted with eq 3 as shown in Figure 2. It is interesting to note that the intercept of the line with temperature $T_{p}$ is in the vicinity of the $\alpha$-relaxation temperature at which the crystalline phases start to flow and the sample is in the quasi-melt state. Thus it follows that the plastic stress associated with the flow process of crystalline parts approaches zero as the temperature is going to $T_{p}$.

The second term $\sigma_{e}$ in eq 1 represents the mechanical response for network deformation. The network elongation is essentially similar to that of a com-

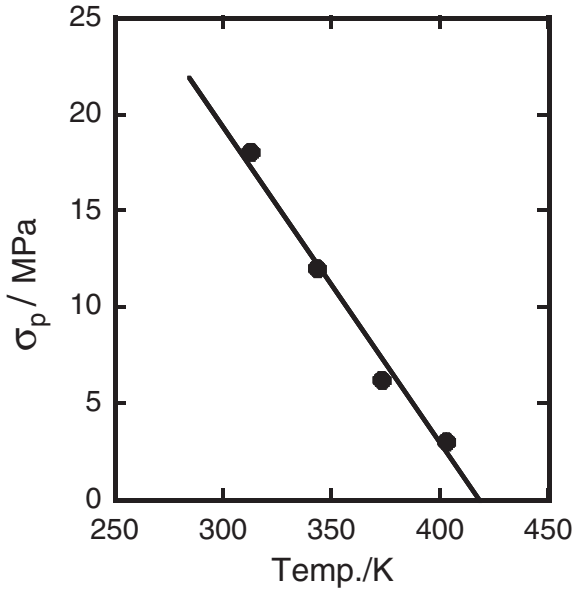

Figure 2. Temperature dependence of stress level at necking region of a polypropylene sample.

pressed gas since the retractive force of a network elastomer and the pressure $P$ of a gas are both primarily entropically derived. ${ }^{32}$ The equation of state for an ideal gas is given by $P=N k T / V$ and the constitutive equation for an ideal Gaussian network is expressed as $\sigma_{e}=N k T e$ in which $k$ is the Boltzmann constant, $N$ is the number of gas molecules or cross-links in a network, and $e$ is the extent of deformation or strain given by $\lambda-\lambda^{-2} \cdot{ }^{23,24}$ Both relations are equivalent when the pressure is replaced by the nominal stress, and the reciprocal volume $(1 / V)$ of an ideal gas is replaced by the extent of deformation as demonstrated by Mark. ${ }^{32}$ It should be noted here that the extent of deformation is given by the deviatoric strain in the case of uniaxially stretched Gaussian network. ${ }^{33}$

Heise-Kilian et al. $^{23}$ showed that the constitutive relation of semicrystalline polymers can be expressed by the extension of the ideal gas model to the van der Waals equation. The constitutive relation of the network component can be obtained in the same manner as presented by the construction of the van der Waals equation:

$$
\sigma_{e}=\frac{N k T e}{1-b e}-a e^{2}
$$

where the parameter $b$ corresponds to the reciprocal of the maximum elongation and the parameter $a$ regards local attractive interactions. According to their analysis for applying the constitutive equation to experimental stress-strain curves of PE samples, the values of $a$ and $b$ are $0.08 G$ and 0.056 for HDPE, and they are $0.13 G$ and 0.05 for LDPE where $G$ is the stresshardening modulus. ${ }^{27}$ The values of $G$ were estimated to be $c a .6 \mathrm{~N} / \mathrm{mm}^{2}$ for HDPE and $c a .3 \mathrm{~N} / \mathrm{mm}^{2}$ for LDPE by reading off from their experimental data. By putting eqs 3 and 4 into eq 1 we obtain the stressstrain relation in the post-yield region as follows: 


$$
\sigma=\gamma\left(T_{p}-T\right)+\frac{N k T e}{1-b e}-a e^{2}
$$

In eq 5 , the parameters $a$ and $b$ will be a weak function of temperature. For the sake of simplicity, we assume here that $a$ and $b$ are independent of temperature. Note that the first term represents the thermoplasticity and the second term represents the entropic elasticity. Let the relative temperature to be $\Theta=T /$ $T_{p}$, and let $\kappa=\gamma T_{p}$ and $\Re=N k T_{p}$. Then the constitutive relation eq 5 can be rewritten as:

$$
\sigma=\kappa(1-\Theta)+\frac{\Re \Theta e}{1-b e}-a e^{2}
$$

Furthermore the constitutive relation eq 6 can be expressed as a function of the cubic relation of $\varepsilon$ using the relation $\sigma_{e}=\sigma-\kappa(1-\Theta)$,

$$
e^{3}-\frac{1}{b} e^{2}+\frac{1}{a}\left(\frac{\Re \Theta}{b}+\sigma_{e}\right) e-\frac{1}{a b} \sigma_{e}=0
$$

Accordingly, the mechanical behavior depends on whether this cubic relation of $e$ has one real root or three real roots depending on each coefficient.

All gases can be liquefied by compressing at a certain temperature. However, there exists a critical temperature above which the gas cannot be liquefied. When a substance is liquefied at a temperature below the critical temperature, the isothermal $P-V$ curve becomes flat. This equilibrium process of transformation from gas to liquid is strikingly similar to that transformation from the isotopic structure to the oriented fibril structure. ${ }^{11}$ The actual force is constant in the equilibrium state that both the oriented (necked) and disoriented (un-necked) phases are coexisted and the unnecked phase is steadily transformed into the necked phase by the external force. In addition, at a higher temperature the materials become too soft to be necked and they homogeneously deform. This also remarkably analogizes with a phase transition between gas and liquid above a critical temperature.

Taking first and second derivatives in eq 7 with respect to $e$ and setting them independently equal to zero, we have the critical strain and critical reduced temperature

$$
e^{*}=\frac{1}{3 b} \quad \text { and } \quad \Theta^{*}=\frac{8 a}{27 b \Re}
$$

and the critical stress is therefore

$$
\sigma_{e}^{*}=\frac{a}{27 b^{2}} \quad \text { or } \quad \sigma^{*}=\kappa\left(1-\Theta^{*}\right)+\frac{a}{27 b^{2}}
$$

It is convenient to normalize the original variables $\left(e, \Theta, \sigma_{e}\right)$ in terms of this critical point; i.e., to introduce new dimensionless state variables. In order to transform eq 7 into a more simple form, let

$$
\begin{aligned}
& x \equiv \frac{e}{e^{*}}-1=3 b e-1 \\
& t \equiv \frac{\Theta}{\Theta^{*}}-1=\frac{27 b \Re}{8 a} \frac{T}{T_{p}}-1 \\
& p \equiv \frac{\sigma_{e}}{\sigma_{e}^{*}}-1=\frac{27 b^{2}}{a}\left(\sigma-\kappa\left(1-T / T_{p}\right)\right)-1
\end{aligned}
$$

Conbining eqs $10-12$ and eq 7 leads to

$$
x^{3}+u x+v=0
$$

where

$$
\begin{aligned}
& u=\frac{1}{3}(8 t+p) \\
& v=\frac{2}{3}(4 t-p)
\end{aligned}
$$

Eq 13 is the resultant constitutive relation with reduced mechanical parameters. In this paper, the dimensionless parameters $x, p$, and $t$ are referred to as the reduced strain, the reduced stress, and the reduced temperature, respectively. The real solutions of this polynomial of $x$ can be controlled by two coefficients of $u$ and $v$. This polynomial is called a cusp catastrophe equation whose canonical form of the universal unfolding with two control parameters of $u$ and $v$.

According to the catastrophe theory ${ }^{34,35}$ as shown in Appendix, if a system is specified by one state variable $x$ and two-rank control space $\mathbf{s}=(v, u)$, the state of the system is described by the following potential function:

$$
U(x ; \mathbf{s})=\frac{1}{4} x^{4}+\frac{1}{2} u x^{2}+v x+U_{0}
$$

where $U_{0}$ is a function of being independent of $x$ and s. Each point in the s-plane gives rise to a potential function $U(x ; \mathbf{s})$ and the state of the system represented by $U(x ; \mathbf{s})$ is determined by a minimum of $U(x ; \mathbf{s})$ with $x$. Consequently, the location of the minima gives the equilibrium surface as $\mathbf{s}$ varies. The equilibrium surface, composed of the set of all equilibrium points, can be derived from the derivative of eq 16 with $x$ :

$$
\frac{\partial U(x ; \mathbf{s})}{\partial x}=0
$$

which leads to eq 13. Accordingly, the reduced constitutive equation eq 13 can be considered to be an equilibrium surface which is given by the minima of the potential function $U(x ; \mathbf{s})$ with two control parameters $u$ and $v$. The control space $\mathbf{s}=(v, u)$ is completely given by two independent parameters of the reduced temperature $t$ and the reduced stress $p$.

When we plotted the fixed $x^{-1}$ above the $\mathbf{s}=(v, u)$ plane, a movement of the state point $\left(v, u, x^{-1}\right)$ according to eq 13 represents the cusp catastrophe described by the equilibrium surface containing fold or pleat 


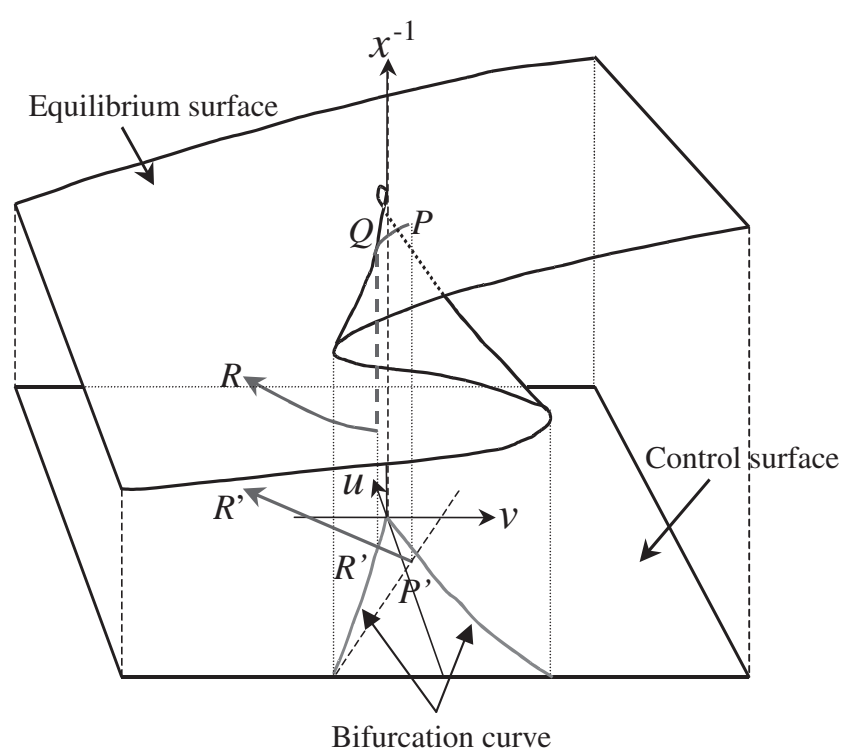

Figure 3. Cusp catastrophe model for necking.

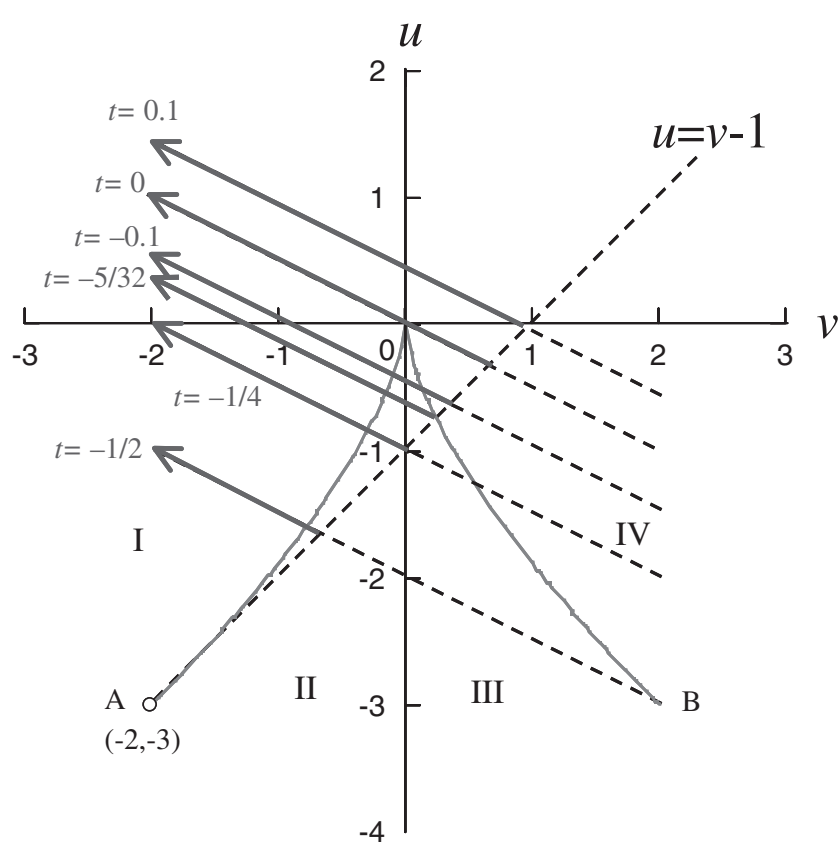

Figure 4. Control space. The red lines denote the bifurcation set and deformation lines at various reduced temperature.

as illustrated in Figure 3. The deformation behavior is described by a movement of the state point $\left(v, u, x^{-1}\right)$ along the equilibrium surface. As shown in Appendix, the bifurcation curves are represented by the projection of these folds onto the control planes $\mathbf{s}=(v, u)$ giving

$$
4 u^{3}+27 v^{2}=0
$$

The bifurcation curve is the line that marks the edges of the pleat in the equilibrium surface, where the top and bottom folds are projected on the left and right bifurcation curves as shown in Figures 3 and 4, respec-

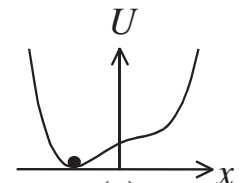

(a)

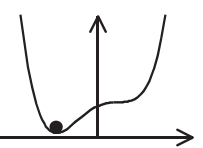

(b)

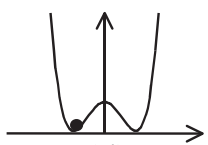

(d)

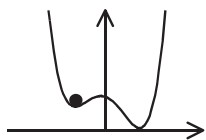

(e)

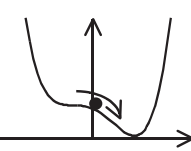

(f)

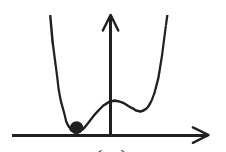

(c)

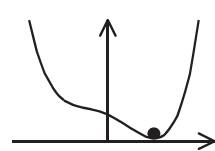

(g)
Figure 5. Variation of the state potential energy for necking process. The small ball denote the location of deformation point. (a) reflects region IV, (b) and (f) on the bifurcation set, (d) on the Maxwell line, (c) in the region III, (e) in the region II, and (g) in the region $\mathrm{I}$.

tively. Therefore, when the control point $(v, u)$ passes through the left branch of the bifurcation curves, the state point on the equilibrium surface encounters the edge of the fold, where a slight change in the parameters causes a fall or jump to the lower equilibrium surface.

The variation of the corresponding potential energy profile for the changes in the control parameters is illustrated in Figure 5. Systems that are driven toward equilibrium values, such as the small particle in Figure 5, can be classified according to their configuration of minimum points. When the control point passes the left bifurcation curve, the local minimum suddenly disappears resulting in that the particle will suddenly move from its old position to a new minimum as illustrated in Figure 5f. This corresponds to the jump to the lower equilibrium surface on the cusp surface. As a consequence the sudden decrease in the $x^{-1}$ value takes place when the control point passes through the left bifurcation curve.

Considering the general conditions that $e \geq 0$, $\sigma_{e} \geq 0$, and $T \geq 0$ in eqs $10-12$, we have the following inequality:

$$
x \geq-1, t \geq-1, p \geq-1
$$

This condition leads to the necessary condition for control space $\mathbf{s}$ :

$$
I I=\left\{(v, u) \in \mathbf{R}^{2} \mid v \geq-2, u \geq-3\right\}
$$

On eliminating $t$ from eqs 14 and 15 , and putting $p=-1$, we have the start points of deformation at any temperature:

$$
u=v-1
$$

It is interesting to note that the left bifurcation curve is 

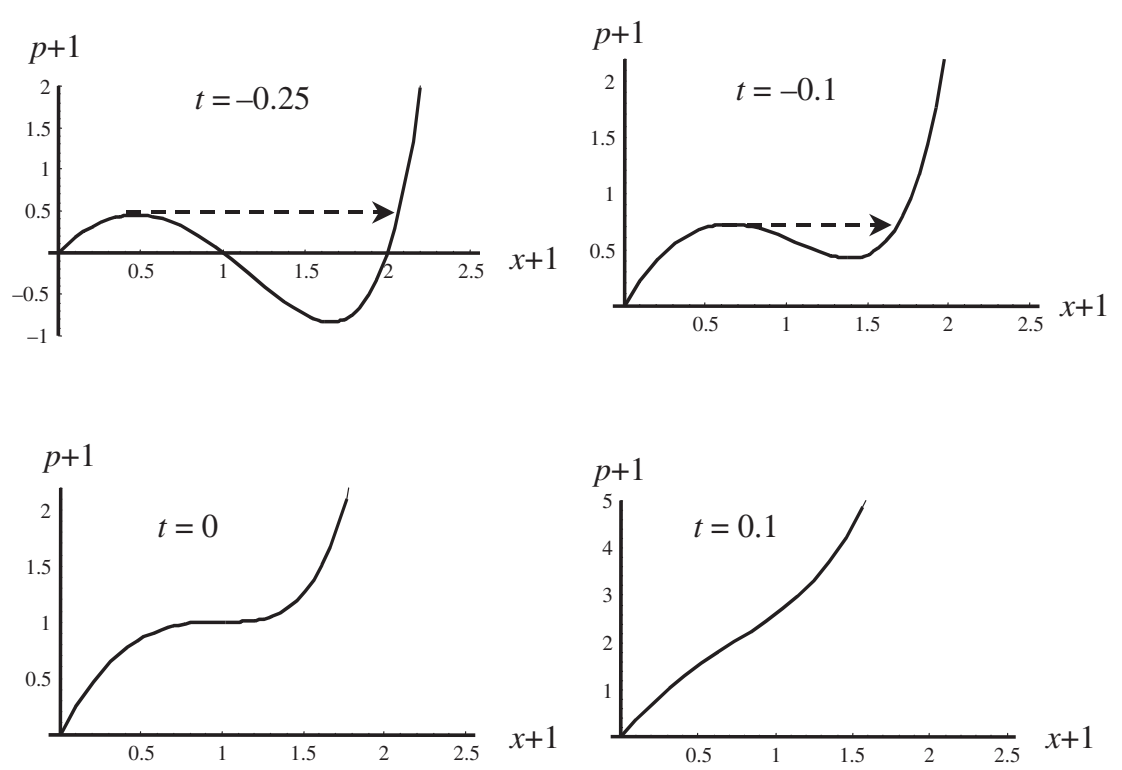

Figure 6. Reduced stress $p+1 v s$ reduced strain $x+1$ at various reduced temperature $t$.

contact with the start line eq 21 at the point $(v, u)=$ $(-2,-3)$. The start line of deformation and the bifurcation curve are also shown in Figure 3. Moreover, on eliminating $p$ from eqs 14 and 15 , we have

$$
u=-\frac{1}{2} v+4 t
$$

When the deformation proceeds at a fixed temperature $t$, the control point $\mathbf{s}=(v, u)$ moves along a straight line. The deformation line eq 22 intersects the start line eq 21 , giving the onset point $(8 t / 3+2 / 3$, $8 t / 3-1 / 3)$ of deformation.

We now return to the basic constitutive equation eq 13. Combination of eqs 14 and 15 with eq 13 gives

$$
p=\frac{3 x^{3}+8 t x+8 t}{2-x}
$$

eqs 23 and 19 require the following condition:

$$
-1 \leq x<2
$$

Figure 6 shows the reduced stress $p$ vs reduced strain $x$ at constant temperature $t$. For $-1 \leq t \leq 0$, the stress $p$ has a maximum and the negative slope in stressstrain curve appears. This part is an unstable region. When a state point passes through the line $v=0$, the stable state changes to the metastable state. At further deformation the metastable state disappears on the bifurcation curve and the state point jumps to the other minima. The disappearance of the original minima corresponds to the maximum point on the stress-strain curve (Figure 5f) so that the strain $x$ jumps along the arrow. Note that for $-1 \leq t \leq-1 / 4$, the start point is in the metastable state (Figure 5e). For $-1 / 4 \leq t \leq-5 / 32$ there are two minima but the start point is the stable state (Figure $5 \mathrm{c}$ ). For $-5 / 32 \leq t \leq 0$ there is one minimum (Figure 5a) and the metastable minima appears (Figure 5c) after passing the right bifurcation line. For high temperature $0 \leq t$, the stress monotonously increases with strain, indicating no structural transformation and an one-to-one correspondence between the stress and the strain. In this region, the deformation homogeneously proceeds without necking. At higher temperatures $(t \geq 0)$, the local necking process will not occur. This corresponds to the fact that the material is too soft to show necking at a higher temperature.

The sudden process leading to a highly oriented phase locally takes place. If the highly oriented or strained phase suddenly appears over the whole specimen, the length of the sample specimen must be instantly elongated to the corresponding length. Considering that the sample length during a deformation gradually increases according to the cross-head speed, the highly oriented phase could not instantly evolve over the whole specimen when the deformation reaches the bifurcation point. Thus this sudden structural transition could not reach the whole specimen so that the highly oriented region is confined in a very local portion of the specimen which will be a seed for necking. Consequently, this highly strained part acts as a neck initiation and further deformation will cause neck formation and propagation according to ColemanNewman theory. ${ }^{16,17}$

The maximum point of $p$ gives the strain $x_{N}$ at which the necking begins. As seen in Figure 7, the numerical value of $x_{N}$ increases with increasing the temperature $t$. This feature also seems to be qualitatively in accordance with the empirical observation that the onset of necking remarkably is delayed by a rise of temperature and/or by softening of materials. 


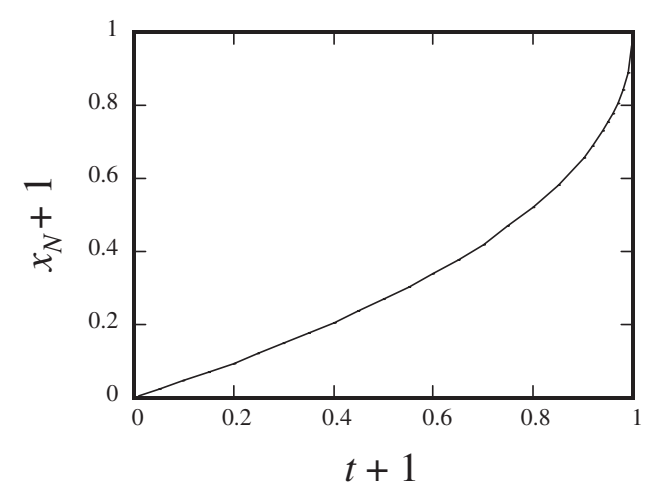

Figure 7. Reduced temperature dependence of reduced strain at the onset of necking.

The horizontal line at the maximum point intersects the stress-strain curve, giving the necking draw ratio. In order to predict the neck draw ratio, we have to know the temperature dependence of the maximum strain $b^{-1}$. Here we does not discuss on the neck draw ratio because of the beyond the scope of this work.

\section{DISCUSSION}

Ericksen ${ }^{11}$ demonstrated a remarkable similarity between the necking and phase transition in van der Waals gas. Using this analogy, he assumed the constant equilibrium force (Maxwell line) which is determined so as to be equal in the energy level of the van der Waals loops. This corresponds to that the lowest of the two minima in potential function is chosen. Therefore, when the state point passes the line $v=0$, the point jump to the lowest minimum. This process is called the "Maxwell convention". On the other hand, that the system will remain in an original minimum until this minimum disappears is called the "delay convention". This leads to a hysteresis phenomenon. If the Maxwell convention is accepted, for $-1 \leq t \leq$ $-1 / 4$ the system has to move to the new minimum at the start point of deformation, indicating that the necking appears before yielding since the yield point is assumed to be the onset point in the present deformation model. In addition, the Maxwell convention has no hysteresis after and before the neck initiation. These results seem to be empirically unacceptable for usual semicrystalline polymers because of the fact that the necking takes place after the engineering stress has passed its maximum value ${ }^{8,9}$ and that the necking process is likely to be accompanied with mechanical hysteresis.

The existence of lamellar clusters and ICLs is theoretically derived by assuming the occurrence of a chain ends-exclusion process during solidification: ${ }^{20,21}$ a structural unit having a single Gaussian chain, the inside of which includes intertwining chains, sponta- neously built up from the melts and the crystallization within the structural units leads to the lamellar clusters. This implies that the lamellar cluster thickness $L_{c}$ is almost equal to the end-to-end distance of unperturbed chains in the melt and is proportional to the square root of molecular weight. Some extended molecules with an end-to-end distance much larger than the thickness of a lamellar cluster have the capability of linking adjacent clusters. These extended molecules will coalesce to form single intercluster links which correspond to the intercrystalline links shown in TEM observation by Keith et al. ${ }^{18,19}$ The probability for forming an ICL can be numerically estimated from the probability that the end-to-end distance of a random coil is in excess of $2 L_{c}$.

The necking phenomena usually appear when a homogeneous semicrystalline material is uniaxially stretched. In this case, two uniform sections appear in the sample; one being nearly equal to initial thickness and another being considerably thinner in transverse dimensions. The experimental results by Keith et al. as well as our theoretical consideration strongly suggest that the ICL units randomly disperse in the spherulitic matrix in the undeformed state. The molecular interactions such as attractive ordering and volume exclusion between ICLs leads to a van der Waals type of constitutive equation such as eq 5 in the same manner as the parameters $a$ and $b$ in usual van der Waals liquids. Consequently, further deformation leads to catastrophic ordering but the ordering is localized (see Figure 1). This local ordering of ICLs results in a slight reduction of the cross-sectional area leading to neck formation and propagation. Therefore the neck initiates in the stress-drop region between the yield point and the onset of the plateau stress region. This is in accord with familiar observation of tensile necking behavior in PE and PP. This strongly demonstrates that amorphous polymers and liquid-crystalline polymers having crystallizable units or orderable ones will be capable of showing the neck phenomena whereas the materials having no ability of aggregation or ordering under a uniaxial elongation will not show the neck.

Consequently, the ICLs support the external force and their tractive forces act on the cluster surface, leading a portion of the lamellar cluster to be bent around one ICL. It follows that Young's modulus is predominantly determined by the bending modulus of lamellar clusters which are governed by the crystallinity or crystalline lamellar fraction within a lamellar cluster. Further deformation leads to splaying of the intercluster regions and bending of the lamellar clusters between link points, and eventually to the fragmentation of lamellar clusters into the structural units. The failure of the lamellar clusters corresponds to the 
yield process so that the magnitude of yield stress depends on the strength of crystalline lamellae; that is the packing of crystal stem or the lamellar thickness, within lamellar clusters units. Since the roots of the ICLs are considered to be embedded into the structural units, the yield process makes it possible to release the constrains of the ICL units and to cause the rearrangement of the ICL units like micro shish-kabab structure. ${ }^{36}$ Thus the attractive and/or repulsive interactions between the released ICL lead to catastrophic alignment. The degree of alignment of the ICL units initiated at catastrophe point determines the neck draw ratio.

The plastic component $\sigma_{p}$ or the yield stress is related to the strength of crystalline lamellae within the lamellar clusters and modulus increases with the rigidity of lamellar clusters relating to the higher crystallinity or the thinner amorphous layers. ${ }^{37,38}$ Whereas the dominant parameters for network component in constitutive relation are the $a$ and $b$ parameters that govern the neck initiation. The parameter $a$ expresses attractive interactions between the ICL units the length of which is related to the molecular weight. When we take the limit $e \rightarrow b^{-1}$, the network stress becomes infinite, indicating that $b^{-1}$ is the maximum extension $e_{m}\left(=\lambda_{m}-\lambda_{m}^{-2}\right)$ of the cluster units between ICLs. Considering that the size of the cluster units corresponds to the end-to-end distance $\left\langle r^{2}\right\rangle^{1 / 2}$ and their maximum extended length corresponds to the ultimate extended chain length, the maximum extension $\lambda_{\mathrm{m}}$ becomes proportional to the square of molecular weight. Consequently, the parameters $a$ and $b^{-1}$ are considered to be positive dependence of molecular weight.

As well known, the external field induces some order in the isotropic phase and a first-order transition from isotropic phase to an ordered nematic phase. The theoretical treatment for the isotropic-nematic transition can be done by using a Landau-de Gennes type expansion of the free energy, as follows: ${ }^{39-41}$

$$
F=\eta Q+\alpha\left(T-T_{c}\right) Q^{2}-\beta Q^{3}+\gamma Q^{4}
$$

where $Q$ is the order parameter, $T_{c}$ is the transition temperature, and $F_{0}$ is the constant. The coefficient $\alpha, \beta, \gamma$, and $\eta$ are dependent on the external field. It is very important to note that this equation can be reduced to the cusp equation eq 13 by canceling the $Q^{3}$ term. This indicates that the onset of nematic-isotropic transition is also one of cusp catastrophe phenomena showing discontinuous and sudden changes in the molecular ordering. This is a very important fact for understanding the onset of necking. The cusp catastrophic ordering of ICLs acts as an initiator of necking and the extent of the catastrophic ordering corresponds to the draw ratio of neck portion. This structural feature seems to be consistent with the experi- mental results of TEM studies on deformed PE films by Kanig, ${ }^{22}$ in which a network of fibers crosslinked by crystal blocks was observed.

\section{FINAL REMARKS}

The molecular interactions such as attractive ordering and volume exclusion between intercrystalline links lead to a van der Waals type of constitutive equation demonstrated by Ericksen ${ }^{11}$ and Kilian et $a l^{23}$ in the same manner as the parameters $a$ and $b$ in usual van der Waals liquids. Consequently, further deformation leads to catastrophic ordering but the ordering is localized (see Figure 1). This local ordering of intercrystalline links or strained portion results in a slight reduction of the cross-sectional area leading to neck formation and propagation. This strongly demonstrates that amorphous polymers and liquid-crystalline polymers with crystallizable units or rod-like molecules having a potential to be ordered during deformation will be capable of showing the neck phenomena whereas the materials having no ability of aggregation or ordering under a uniaxial elongation will not show the neck. In other words, the necessary and sufficient conditions leading to the occurrence of neck is that there exists the intercrystalline links or rod-like molecules as high-ordered structure or that strain-induced crystallization or molecular ordering takes place during stretching.

Mechanical instability in metals and rocks has been recently analyzed by using the catastrophe theory. ${ }^{35}$ It was demonstrated that mechanical buckling of metals, ${ }^{42}$ rock-sliding process, ${ }^{43}$ stress-induced crystal modification and shape-memory effects of metals and alloys ${ }^{44}$ can be analyzed on the basic of the catastrophe theory. These studies suggest that other important problems in mechanical instability of polymers such as stress-induced crystallization behavior, neck-flow transition of fibers, and melt fracture in polymer processing could be qualitatively understood by the catastrophe theory. The catastrophe theory potentially will be a powerful new tool for providing the physical insight into various mechanical problems of polymeric materials.

\section{APPENDIX}

Catastrophe theory is a mathematical technique developed by Thom $^{34}$ for modeling natural phenomena, which contain discontinuous and sudden changes in the values of one or some parameters. This theory has been applied in many fields such as geology, mechanics, physics, chemical reaction, economics, and social science. ${ }^{45-47}$ Thus it mathematically describes the sudden changes caused by small and continuous 
changes in control parameters which are independent variables that influence the state. The phase transition and transformation are typical catastrophe phenomena. ${ }^{44,47}$ According to Thom's elementary catastrophe theory, if a system is specified by one state variable $x$ and $k$-rank control space $\mathbf{s}=\left(s_{1}, s_{2}, \ldots, s_{\mathrm{k}}\right)$, the state of the system is specified by the following function:

$$
\begin{aligned}
& U\left(x ; s_{1}, s_{2}, \ldots s_{k}\right) \\
& \quad=g_{0}(x)+s_{1} g_{1}(x)+s_{2} g_{2}(x)+\ldots+s_{k} g_{k}(x)
\end{aligned}
$$

The function $U(x ; \mathbf{s})$ acts precisely as a potential function. If the control space is given by two-rank space, the potential function can be given by

$$
U(x ; \mathbf{s})=s_{4} x^{4}+s_{3} x^{3}+s_{2} x^{2}+s_{1} x+s_{0}
$$

which can be rewritten as eq 16 by the shift of origin of $x$ and its reduction. Consequently, the equilibrium surface is given by

$$
M_{F}=\left\{(x ; v, u) \in \mathbf{R}^{3} \mid \frac{\partial U}{\partial x}=x^{3}+u x+v=0\right\} \text { (A.3) }
$$

When we plot the fixed $x^{-1}$ above the $\mathbf{s}=(v, u)$ plane, we get the cusp surface which holds over on itself in certain places as shown in Figure 3. A fold or pleat curve is most naturally viewed as a section through the cusp catastrophe surface, passing transversely to the cusp axis $x^{-1}$. The behavior of the system occurs as a smooth or catastrophic movement of the state point $\left(v, u, x^{-1}\right)$ along the equilibrium surface. The control plane is the surface defined by projection of a three-dimensional equilibrium surface to a two-dimensional control space. The projection of these folds onto the control plane $\mathbf{s}=(v, u)$ yields the bifurcation curves (see Figure 4). By combining $\partial U / \partial x=0$ and $\partial^{2} U / \partial x^{2}=0$, the bifurcation set can be given by

$$
B_{F}=\left\{(v, u) \in \mathbf{R}^{2} \mid 4 u^{3}+27 v^{2}=0\right\}
$$

The changes in the control plane $\mathbf{s}=(v, u)$ gives rise to two different types of potential function: if $\mathbf{s}$ lies outside the bifurcation curve $4 u^{3}+27 v^{2}=0$, the potential function $U(x ; \mathbf{s})$ has one minimum; if $\mathbf{s}$ lies inside the cusp curve, $U(x ; \mathbf{s})$ has two minima. The bifurcation set defines the thresholds where sudden changes can take place. As long as the state of the system remains out side the bifurcation set, the behavior varies smoothly and continuously as a function of control parameters. Even on entering the bifurcation set, no abrupt change is observed. When the control point $(v, u)$ passes all the way through the bifurcation curve, however, a catastrophe is inevitable. For example, if a material undergoes deformation and the control point moves along the path $P^{\prime} \rightarrow Q^{\prime} \rightarrow R^{\prime}$ shown in Figure 3, then the state of the system can be carried over the edge of the upper surface, after which it drops discontinuously to the lower surface as shown by the path $P \rightarrow Q \rightarrow R$ on Figure 3. When the deformation proceeds and the control point to pass through the left bifurcation curve, the value of $x^{-1}$ suddenly drops on the lower equilibrium state. This jump is associated with the neck initiation in the text.

The control space can be divided into four regions, as illustrated in Figure 4. In the figure, the left and right branches of the bifurcation set are shown in $\mathrm{OA}$ and $\mathrm{OB}$ curves, respectively. When the control point is in the region I or IV, the potential function $U(x ; \mathbf{s})$ has only one real root, corresponding to a minimum values of the potential energy as shown in Figure $5 \mathrm{~g}$ or $5 \mathrm{a}$, respectively and this takes only one stable state of the system. When the control point is in the region II or III, $U(x ; \mathbf{s})$ has three different real roots, corresponding to two local minimums and one maximum points as shown in Figure $5 \mathrm{e}$ or $5 \mathrm{c}$. When the control point is on the bifurcation curve, $U(x ; \mathbf{s})$ has one minimum and one inflection point (see Figure $5 \mathrm{~b}$ or $5 \mathrm{f}$ ). When the control point is on the line of $v=0, U(x ; \mathbf{s})$ has symmetric double wells (see Figure 5d).

For $-1 \leq t \leq-1 / 4$ the system initially locates on the metastable minimum. For example, this corresponds to the line denoted by $t=-1 / 2$ in Figure 4 . When the deformation proceeds and the control point to pass through the left bifurcation curve, the value of $x^{-1}$ suddenly drops on the lower equilibrium state. This corresponds to the changes of the potential energy; (e) $\rightarrow$ (f) $\rightarrow$ (g). The drop of $x^{-1}$ indicates the sudden increase in strain, i.e., the initiation of local cold-drawing. If $-1 / 4 \leq t \leq-5 / 32$, the deformation leads the potential energy profile to change (c) $\rightarrow$ (d) $\rightarrow$ (e) $\rightarrow$ (f) $\rightarrow$ (g). If $-5 / 32 \leq t \leq 0$, the deformation leads the potential energy profile to change from (a) to (g), see e.g. the line $t=-0.1$ in Figure 4. Similarly when the control point passes the left bifurcation curve, the sudden increase in strain $x$ take places. For $t \geq 0$, the state point moves smoothly on the equilibrium surface resulting in that there are no catastrophic jump. The case of $t=0$ corresponds to the critical point.

Acknowledgment. This work is partially supported by a grant-in-aid for scientific research from Ministry of Education, Culture, Sports, Science and Technology.

\section{REFERENCES}

1. W. H. Carothers and W. J. Hill, J. Am. Chem. Soc., 54, 1579 (1932).

2. A. Chudnovsky and S. Preston, Mecha. Res. Commun., 29, 
465 (2002).

3. P. Brauwer and F. H. Müller, Kolloid Z., 135, 65 (1954).

4. Y. S. Lazurkin, J. Polym. Sci., 30, 595 (1958).

5. P. I. Vincent, Polymer, 1, 7 (1960).

6. S. W. Allison and I. M. Ward, Br. J. Appl. Phys., 18, 1151 (1967).

7. G. R. Strobl, "The Physics of Polymers," Springer-Verlag, Berlin, 1998, p 359.

8. N. W. Brooks, R. A. Duckett, and I. M. Ward, Polymer, 33, 1872 (1992).

9. B. Crist and C. Metaxas, J. Polym. Sci., Part B: Polym. Phys., 42, 2081 (2004)

10. J. W. Hutchinson and K. W. Neale, J. Mech. Phys. Solids, 31, 405 (1983).

11. J. J. Ericksen, J. Elasticity, 5, 191 (1975).

12. B. Bernstein and L. J. Zappas, J. Rheol., 25, 83 (1981).

13. A. I. Leonov, Int. J. Solids Struct., 39, 5913 (2002).

14. A. N. Gent and J. Jeong, Polym. Eng. Sci., 26, 285 (1986).

15. A. N. Gent and S. Madan, J. Polym. Sci., Part B: Polym. Phys., 27, 1529 (1989).

16. B. D. Coleman and D. C. Newman, J. Appl. Polym. Sci., 45, 997 (1992).

17. B. D. Coleman and D. C. Newman, J. Polym. Sci., Part B: Polym. Phys., 30, 25 (1992).

18. H. D. Keith, F. J. Padden, and R. G. Vadimsky, J. Polym. Sci., A-2, 4, 267 (1966).

19. R. G. Vadimsky, H. D. Keith, and F. J. Padden, J. Polym. Sci., A-2, 7, 1367 (1969).

20. M. Takayanagi, K. Nitta, and O. Kojima, J. Macromol. Sci., Part B: Phys., B42, 1049 (2003).

21. K. Nitta and M. Takayanagi, J. Macromol. Sci., Part B: Phys., B42, 107 (2003).

22. G. Kanig, J. Cryst. Growth, 48, 303 (1980).

23. B. Heise, H.-G. Killian, and W. Wulff, Prog. Colloid Polym. Sci., 67, 143 (1980).

24. H.-G. Kilian, Polymer, 19, 664 (1978).

25. H.-G. Kilian, Colloid Polym. Sci., 262, 374 (1984).
26. C. G'Cell and J. J. Jonas, J. Mater. Sci., 16, 1956 (1981).

27. R. N. Haward, Polymer, 28, 1485 (1987).

28. Y. Song, K. Nitta, and N. Nemoto, Macromolecules, 36, 8066 (2003).

29. H. Eyring, J. Chem. Phys., 4, 283 (1936).

30. C. Bauwens-crowet, J. M. Ots, and J. C. Bauwens, J. Mater. Sci., 14, 583 (1974).

31. S. H. Joseph, J. Polym. Sci., 16, 1971 (1978).

32. J. E. Mark, in "Physical Properties of Polymers," 2nd ed., ACS, Washington DC, 1993, Chap. 1, p 3.

33. H.-G. Kilian, Polymer, 22, 209 (1981).

34. R. Thom, "Structural Stability and MorphologenesisAn Outline of a General Theory of Models," Benjamin/ Cummings, London, 1975.

35. T. Poston and I. Stewart, "Catastrophe theory and its applications," Pitman Pub. 1978.

36. P. J. Barham, in "Structure and Properties of Oriented Polymers" 2nd ed. I. M. Ward, Ed., Chapman \& Hall, 1997, Chap. 3, p 153.

37. K. Nitta and N. Yamaguchi, Polym. J., 38, 122 (2006).

38. K. Nitta, Comput. Theor. Polym. Sci., 9, 19 (1999).

39. M. Warner, K. P. Gelling, and T. A. Vilgis, J. Chem. Phys., 88, 4008 (1988).

40. M. Warner and X. J. Wang, Macromolecules, 24, 4932 (1991).

41. P. Maissa, Y. Lansac, and F. Fried, Polymer, 37, 2039 (1996).

42. X. Lignos, G. Ioannidis, and A. N. Kounalds, Int. J. Nonlinear Mech., 38, 1163 (2002).

43. S. Qin, J. J. Jiao, and S. Wang, Rock Mech. Rock Eng., 34, 119 (2001).

44. F. Falk, Arch. Mech., 35, 63 (1983).

45. R. Rosen, Landscape Ecology, 3, 207 (1989).

46. J. Thompson and G. W. Hunt, J. Appl. Math. Phys., 26, 581 (1975).

47. J. Gaite, J. Margalef-Roig, and S. Miret-Artes, Phys. Rev. B: Condens. Matter Mater. Phys., 57, 13527 (1998). 